\title{
BMJ Open Standardised versus individualised multiherb Chinese herbal medicine for oligomenorrhoea and amenorrhoea in polycystic ovary syndrome: a randomised feasibility and pilot study in the UK
}

\author{
Lily Lai, ${ }^{1}$ Andrew Flower, ${ }^{1}$ Philip Prescott, ${ }^{2}$ Trevor Wing, ${ }^{3}$ Michael Moore, ${ }^{1}$ \\ George Lewith ${ }^{1}$
}

To cite: Lai L, Flower $A$, Prescott $P$, et al. Standardised versus individualised multiherb Chinese herbal medicine for oligomenorrhoea and amenorrhoea in polycystic ovary syndrome:

a randomised feasibility and pilot study in the UK. BMJ Open 2017;7:e011709. doi:10.1136/bmjopen-2016011709

- Prepublication history and additional material is available. To view please visit the journal (http://dx.doi.org/ 10.1136/bmjopen-2016011709).

Received 29 February 2016 Revised 22 November 2016 Accepted 12 January 2017

CrossMark

For numbered affiliations see end of article.

Correspondence to

Dr Lily Lai;

L.Y.W.Lai@southampton.ac.uk

\section{ABSTRACT}

Objectives: To explore feasibility of a randomised study using standardised or individualised multiherb Chinese herbal medicine (CHM) for oligomenorrhoea and amenorrhoea in women with polycystic ovary syndrome (PCOS), to pilot study methods and to obtain clinical data to support sample size calculations.

Design: Prospective, pragmatic, randomised feasibility and pilot study with participant and practitioner blinding.

Setting: 2 private herbal practices in the UK. Participants: 40 women diagnosed with PCOS and oligomenorrhoea or amenorrhoea following Rotterdam criteria.

Intervention: 6 months of either standardised CHM or individualised CHM, $16 \mathrm{~g}$ daily taken orally as a tea.

Main outcome measures: Our primary objective was to determine whether oligomenorrhoea and amenorrhoea were appropriate as the primary outcome measures for the main study. Estimates of treatment effects were obtained for menstrual rate, body mass index (BMI), weight and hirsutism. Data were collected regarding safety, feasibility and acceptability.

Results: Of the 40 participants recruited, $29(72.5 \%)$ completed the study. The most frequently cited symptoms of concern were hirsutism, weight and menstrual irregularity. Statistically significant improvements in menstrual rates were found at 6 months within group for both standardised $\mathrm{CHM}$ (mean difference (MD) $0.18 \pm 0.06,95 \% \mathrm{Cl} 0.06$ to $0.29 ; p=0.0027$ ) and individualised CHM (MD 0.27 $\pm 0.06,95 \% \mathrm{Cl} 0.15$ to $0.39 ; p<0.001$ ), though not between group ( $p=0.26)$. No improvements were observed for BMI nor for weight in either group. Improvements in hirsutism scores found within group for both groups were not statistically significant between group ( $p=0.09)$. Liver and kidney function and adverse events data were largely normal. Participant feedback suggests changing to tablet administration could facilitate adherence.

\section{Strengths and limitations of this study}

- This is the first study to evaluate multiherb Chinese herbal medicines in the UK for oligomenorrhoea and amenorrhoea in polycystic ovary syndrome.

- We compared standardised and individualised Chinese herbal medicines which were administered for 6 months.

- We prospectively collected data on safety and adverse events, menstrual regularity, hirsutism, anthropometrics and quality of life.

- We established feasibility of study procedures including recruitment, pharmacy randomisation, questionnaire administration, practitioner blinding and collecting adherence data.

- This feasibility study does not include a control group but which will be incorporated in a definitive trial in primary care.

Conclusions: A CHM randomised controlled trial for PCOS is feasible and preliminary data suggest that both individualised and standardised multiherb CHMs have similar safety profiles and clinical effects on promoting menstrual regularity. These data will inform the design of a study in primary care that will incorporate an appropriate control.

Trial registration number: ISRCTN 31072075 ; Results.

\section{INTRODUCTION}

Polycystic ovary syndrome (PCOS) affects an estimated $6-18 \%$ of women of reproductive age and is a heterogeneous condition characterised by endocrine and metabolic disturbances. $^{1-3}$ Menstrual disturbances are 
common in PCOS, with oligomenorrhoea found in $47 \%$ of adults with PCOS and amenorrhoea in 19.2\%. ${ }^{4}$ Although the pathogenesis of PCOS is complex, it is typically associated with hyperandrogenism, hyperinsulinaemia and an elevated ratio of the gonadotropins luteinising hormone to follicle-stimulating hormone. ${ }^{3}$ Primary care management of menstrual disturbances typically involves oral contraceptives and insulin-sensitising agents but there is evidence of patient dissatisfaction associated with intolerable side effects, consequent poor adherence and potential increase in cardiovascular and metabolic risk. ${ }^{6-10}$ This highlights issues and barriers with current management and warrants exploration of other treatments.

Complementary medicines such as multiherb Chinese herbal medicines (CHMs) are reportedly used by over $70 \%$ of women with PCOS and is a commonly encountered condition among herbal practitioners. ${ }^{11}{ }^{12}$ Previous studies have demonstrated the potential for certain herbs for PCOS through mechanisms such as increasing granulosa production of oestradiol and progesterone, increasing aromatisation of testosterone to $17-\beta$ oestradiol and reducing levels of luteinising hormone. ${ }^{13-15}$ There is also emerging evidence from randomised controlled trials (RCTs) conducted in China and a pilot study conducted recently in the USA highlights the potential for CHM in the management of symptoms such as oligomenorrhoea and amenorrhoea. ${ }^{16-20}$ Although this provides encouraging preliminary evidence, the majority of the RCTs have been conducted in China and are methodologically poor, limiting the generalisability of these findings. This necessitates further exploration of CHM for PCOS within a fully powered RCT in UK primary care.

Given the novelty of offering CHM in UK primary care, it is important to first conduct a feasibility study to reduce uncertainties and facilitate planning of a main study. ${ }^{21-24}$ The aims of this study were to evaluate the feasibility of a randomised study exploring the effects of standardised or individualised multiherb CHM for PCOS-related oligomenorrhoea and amenorrhoea, to pilot study processes and to obtain safety and clinical data to facilitate sample size calculations for a main study.

\section{MATERIALS AND METHODS \\ Design \\ Design and setting}

Details of the study design are provided in our protocol publication. ${ }^{25}$ To summarise our methods, our primary feasibility research question was:

1. Is oligomenorrhoea and amenorrhoea appropriate as the primary outcome measure for the main study?

Our secondary feasibility questions were:

2. Are other measures more appropriate for investigation as the primary outcome measure for the main study?
3. What is the safety profile of CHM?

4. How should the CHM intervention in the main study be delivered?

5. Can a double-blind, randomised trial with CHM for PCOS be conducted?

This was a prospective, multicentre, pragmatic study in private CHM practices in the UK. Participants were randomised to one of two parallel arms comparing standardised multiherb CHM treatment against individualised multiherb CHM treatment. Although individualised CHM is regarded as 'gold standard' by the clinical literature $^{26}$ and by an earlier Delphi study we conducted, ${ }^{27}$ this has limited application in UK primary care. The comparison between standardised and individualised CHM is therefore important to inform the final trial design and to maximise model validity for CHM practice.

Recruitment took place in the community inviting selfreferrals by displaying ethics-approved posters and posts in community pharmacies, community noticeboards, online forums, press advertising and social media. Participants were included if they ${ }^{1}$ were women aged $18-44,{ }^{2}$ presented with oligomenorrhoea or amenorrhoea and ${ }^{3}$ received a diagnosis of PCOS consistent with Rotterdam criteria. ${ }^{28}$ Women were excluded if they ${ }^{1}$ presented with other causes of hyperandrogenism or menstrual irregularities, ${ }^{2}$ were currently or suspected to be pregnant, or actively trying to conceive, ${ }^{3}$ had been breast feeding in the past 6 months, ${ }^{4}$ were receiving prohibited treatments such as hormonal contraceptives, ${ }^{5}$ had a history of liver or kidney pathologies, ${ }^{6}$ had a history of psychotic illness or eating disorders, ${ }^{7}$ had currently active major depression, ${ }^{8}$ were at risk of harmful and hazardous drinking, ${ }^{9}$ reported known allergies to herbal ingredients within standardised $\mathrm{CHM},{ }^{10}$ did not possess spoken or written language skills necessary to participate, ${ }^{11}$ were unable to attend proposed study visits, ${ }^{12}$ presented with abnormal liver and/or kidney function at screening. Diagnosis of PCOS was confirmed through participants providing medical letters and reports from previous medical investigations. Where a diagnosis remained unclear, participants were offered further blood tests and ultrasonography at a private clinic to confirm eligibility. On randomisation and with permission from each participant, their primary care provider was contacted in writing notifying them of their patient's study participation and of the results of any further investigations carried out as part of eligibility assessment.

We required a sample size of 40 , based on requiring data from 15 participants per arm to calculate estimates of treatment effect and associated variability to inform the power calculation for a main study. We accounted for a $25 \%$ dropout rate based on similar studies. ${ }^{29} 30$

Both CHM treatments were dispensed by Phoenix Medical UK and which supplied granulated extracts manufactured in China by Jiangyin Tianjiang Pharmaceutical Company Limited, a certified good 
manufacturing practice company. Participants were prescribed a dose of $16 \mathrm{~g}$ of granulated extracts per day for 6 months and were asked to prepare the granulated extracts twice a day by reconstituting $8 \mathrm{~g}$ of granulated extracts each time with hot water and taking it as a tea. This dose and length of treatment had been informed by our Delphi study. ${ }^{27}$ Our standardised CHM prescription was developed by this research team and was informed by common CHM treatment strategies highlighted by practitioners in our Delphi study. ${ }^{27}$ This prescription was standardised to contain 14 CHMs commonly used in PCOS, the contents of which can be found in table 1. In Chinese medicine terms based on traditional use, this prescription is directed at moving stagnant liver $\mathrm{Qi}$, tonifying the kidney yang and on nourishing and moving the blood. It is not licensed as a proprietary product in the UK but, like individualised prescriptions, is available following a one-to-one consultation with a CHM practitioner in the UK. CHM practitioners in the UK must show evidence of an adequate level of education and training from an accredited institution, or equivalent experience, in order to be registered with a professional organisation such as the Register of Chinese Herbal Medicine (RCHM) or Association of Traditional Chinese Medicine (ATCM). Individualised CHM prescriptions permitted practitioners to prescribe treatment as usual from a range of 270 individual CHMs, and the details of the 20 most commonly prescribed herbs in the individualised CHM group have been provided as an online supplementary data file. No further training regarding individualised CHM prescribing was provided by the study team. Details regarding qualitative testing and characteristics of the granulated extracts have been provided previously. ${ }^{25}$

CHM treatment was offered for 6 months by a practitioner who needed to ${ }^{1}$ have a minimum 5 years in practice, ${ }^{2}$ be registered with a professional CHM organisation in the $\mathrm{UK},{ }^{3}$ be fully insured, ${ }^{4}$ have access to liver and kidney function testing, and ${ }^{5}$ agree to conduct procedures in the protocol. Dietary and lifestyle advice was permitted as part of usual care. Participants were blinded in that they were informed they would be randomised to one of two CHM treatments and were provided no further information regarding treatment differences. An independent statistician (PP) used computergenerated random numbers with allocation ratio $1: 1$ to provide an irregular block allocation sequence. Allocation codes were transferred to sealed opaque envelopes and provided to an RCHM-approved herbal dispensary who conducted randomisation. As practitioners were providing care as well as conducting

Table 1 Contents of standardised Chinese herbal medicine prescription

\begin{tabular}{|c|c|c|c|c|c|c|}
\hline $\begin{array}{l}\text { Chinese } \\
\text { Pinyin name }\end{array}$ & Common name & Family name & Part used & Botanical name & $\begin{array}{l}\text { Dried } \\
\text { herbal daily } \\
\text { dosage }(\mathrm{g})\end{array}$ & Percentage \\
\hline $\begin{array}{l}\text { Bai Shao } \\
\text { (Chao) }\end{array}$ & Peony (dry fried) & Paeoniaceae & Root & Paeonia lactiflora Pall. & 15 & 10.64 \\
\hline Chai Hu & Bupleurum & Umbelliferae & Root & $\begin{array}{l}\text { Bupleurum chinense } \\
\text { DC. }\end{array}$ & 9 & 6.38 \\
\hline Chen $\mathrm{Pi}$ & Tangerine peel & Rutaceae & Peel & $\begin{array}{l}\text { Citrus reticulatata } \\
\text { Blanco }\end{array}$ & 9 & 6.38 \\
\hline Chuan Xiong & $\begin{array}{l}\text { Szechwan lovage } \\
\text { rhizome }\end{array}$ & Umbelliferae & Rhizome & $\begin{array}{l}\text { Ligusticum chuanxiong } \\
\text { Hort. }\end{array}$ & 9 & 6.38 \\
\hline Dang Gui Wei & Angelica extremitas & Umbelliferae & Root tail & $\begin{array}{l}\text { Angelica sinensis } \\
\text { (Oliv.) Diels }\end{array}$ & 9 & 6.38 \\
\hline $\begin{array}{l}\text { Gan Cao } \\
\text { (Mi Zhi) }\end{array}$ & $\begin{array}{l}\text { Liquorice root } \\
\text { (honey-fried) }\end{array}$ & Fabaceae & Root & $\begin{array}{l}\text { Glycyrrhiza uralensis } \\
\text { Fisch. }\end{array}$ & 6 & 4.26 \\
\hline Gou Qi Zi & Goji berry & Solanaceae & Fruit & Lycium barbarum L. & 9 & 6.38 \\
\hline Gui Zhi & Cinnamon twig & Lauraceae & Twig & $\begin{array}{l}\text { Cinnamomum cassia } \\
\text { Presl. }\end{array}$ & 9 & 6.38 \\
\hline Hong Hua & Safflower & Asteraceae & Flower & Carthamus tinctorius L. & 9 & 6.38 \\
\hline Tao Ren & Peach kernel & Rosaceae & Seed & $\begin{array}{l}\text { Prunus persica (L.) } \\
\text { Batsch. }\end{array}$ & 9 & 6.38 \\
\hline Tu Si Zi & Chinese dodder seed & Convolvulaceae & Seed & $\begin{array}{l}\text { Cuscuta chinensis } \\
\text { Lam. }\end{array}$ & 12 & $8.52^{*}$ \\
\hline $\begin{array}{l}\text { Xiang Fu } \\
\text { (Cu Zhi) }\end{array}$ & $\begin{array}{l}\text { Purple nutsedge } \\
\text { (vinegar-fried) }\end{array}$ & Cyperaceae & Rhizome & Cyperus rotundus L. & 12 & $8.52^{\star}$ \\
\hline Yi Mu Cao & Motherwort & Lamiaceae & Top & $\begin{array}{l}\text { Leonurus japonicus } \\
\text { Houtt. }\end{array}$ & 15 & 10.64 \\
\hline Zhi Ke & Bitter orange & Rutaceae & $\begin{array}{l}\text { Mature fruit } \\
\text { Total prescrip }\end{array}$ & $\begin{array}{l}\text { Citrus aurantium L. } \\
\text { tion weight }\end{array}$ & $\begin{array}{r}9 \\
141\end{array}$ & 6.38 \\
\hline
\end{tabular}


objective outcome assessments, it was especially important to this study that practitioner and assessor blinding was maintained. In order to carry this out, practitioners were asked to formulate an individualised prescription for each participant at each visit. This was sent to the herbal dispensary who conducted randomisation at first visit, and dispensed either the standardised or individualised prescription according to randomisation. Prior to and during the study, practitioners and the study team did not have knowledge of the randomisation sequence or of treatment allocation. Participant blinding was not evaluated since participants were not informed regarding the differences between the two CHM treatments. Statistical analysis was conducted blinded.

Our feasibility outcomes were recruitment and retention rates. We included adherence as a part of our feasibility assessment, measured by Morisky Medication Adherence Scale (MMAS) at weeks 4 and at final visit, and by weighing prescription containers at week 12 and at final visit. Practitioner blinding was evaluated through a questionnaire used in previous studies of similar nature and analysed using Bang Blinding Index (BBI) administered at weeks 4,12 and at final visit. ${ }^{31}$ We evaluated participant feedback at final visit and case report form (CRF) data were collected throughout the study as part of usual research practice and analysed to explore areas of potential improvement. At end of study, we conducted an audit of randomisation and allocation procedures to assess security of these processes.

Our primary outcome measure was menstrual regularity; secondary measures were hirsutism using validated modified Ferriman-Gallwey (mFG) questionnaire, anthropometrics using body mass index (BMI), weight, waist circumference, waist-to-hip ratio (WHR) and quality of life questionnaires Polycystic Ovary Syndrome Questionnaire (PCOSQ), Measure Yourself Medical Outcome Profile (MYMOP) and Dermatology Life Quality Index (DLQI). Additional information regarding the specific measures we used and clinical relevance of the individual scales and scores used are available in our study protocol. ${ }^{25}$ Although menstrual regulation is regarded as clinically important, we wished to explore menstrual regulation as the primary outcome for the main study because we remained uncertain regarding the effects of a 6-month CHM treatment on menstruation. We therefore collected clinical data on menstruation to obtain an estimate of treatment effect and associated variability to support sample size calculations for a future study. The appropriateness of menstrual regulation as a primary outcome for the main study was examined by seeing whether or not we could collect menstrual data for analysis. Furthermore, we explored whether menstrual regulation appeared to be an important outcome to participants by ranking patientgenerated MYMOP data whereby participants specified up to two key symptoms (MYMOP1 and optionally MYMOP2) that were of greatest concern to them and which did not have to be PCOS-related. To investigate whether other outcome measures such as $\mathrm{mFG}$ or anthropometrics could be more appropriate as the primary outcome for the main study, we again collected data on treatment effects and where these symptoms ranked in MYMOP to indicate importance to participants.

Outcome measures were taken at baseline and at final visit with the exception of menstrual frequency which was recorded throughout study participation. Objective measures were assessed by the practitioner, and subjective measures were completed by participants in the absence of the practitioner. Liver and kidney function were assessed using alanine aminotransferase (ALT) and creatinine at baseline, week 4 and at final visit.

Our statistical analysis has been published previously in our study protocol. ${ }^{25}$ We planned to pilot statistical analysis in this feasibility study. Continuous variable comparisons were assessed at end of study adjusted for baseline assessments for the two groups using analysis of variance, or an analysis of covariance (ANCOVA) to include factors such as demographic variables. Categorical data were analysed using $\chi^{2}$ tests. Where assumptions of normality were not met, non-parametric methods were used. Since no formal power calculations have been carried out, results from the statistical analysis should be considered preliminary. Textual data collected from sources such as clinical notes and feedback questionnaires were analysed using thematic analysis.

This trial was registered with Current Controlled Trials (ISRCTN 31072075) prior to recruitment.

\section{RESULTS}

\section{Recruitment and study participation}

Three practitioners responded to our request for volunteer for this study, but were unable to assist due to lacking access to safety testing $(\mathrm{n}=1)$ and lacking time $(n=2)$. One practitioner, the main author (LL), provided all treatment from two sites in London and Hertfordshire.

We recruited 40 participants from 245 enquiries (16.3\% eligibility rate) between January 2013 and July 2013; $28(70 \%)$ in London, and $12(30 \%)$ in Hertfordshire. The last participant visit was in February 2014. The most common reasons for ineligibility were receiving prohibited treatment $(n=34)$, having regular periods $(n=27)$ and distance to sites $(n=22)$. Treatment groups were comparable at baseline for demographics (table 2) and outcome measures. Previous use of conventional medication was high in both groups (standardised group $\mathrm{n}=16,80 \%$; individualised group $\mathrm{n}=11$, $55 \%)$, as was previous use of complementary and alternative medicine (CAM; standardised group $\mathrm{n}=14,70 \%$; individualised group $\mathrm{n}=16,80 \%$ ) and previous use of acupuncture or CHM (standardised group $\mathrm{n}=8,40 \%$; individualised group $\mathrm{n}=12,60 \%$ ).

Our participant flow diagram (figure 1) shows that 29 participants $(72.5 \%)$ completed the 6-month treatment, 
Table 2 Baseline demographics for participants

\begin{tabular}{|c|c|c|}
\hline Baseline demographics, n (\%) unless specified otherwise & Standardised $(n=20)$ & Individualised $(\mathrm{n}=20)$ \\
\hline Age, mean (SD) & $28.5(6.0)$ & $30.4(6.6)$ \\
\hline Years since diagnosis, mean (SD) & $7.1(4.8)$ & $9.3(6.7)$ \\
\hline \multicolumn{3}{|l|}{ Menstrual presentation } \\
\hline Oligomenorrhoea & $15(75)$ & $17(85)$ \\
\hline Amenorrhoea & $5(25)$ & $3(15)$ \\
\hline \multicolumn{3}{|l|}{ Current conventional medication use } \\
\hline Yes & $1(5)$ & $2(10)$ \\
\hline No & $19(95)$ & $18(90)$ \\
\hline Of which previously used conventional medication* & $16(80)$ & $11(55)$ \\
\hline \multicolumn{3}{|l|}{ Prior use of } \\
\hline CAM: yes & $14(70)$ & $16(80)$ \\
\hline Acupuncture or CHM: yes & $8(40)$ & $12(60)$ \\
\hline \multicolumn{3}{|l|}{ Parity $\geq 1$} \\
\hline Yes & $4(20)$ & $1(5)$ \\
\hline No & $16(80)$ & $19(95)$ \\
\hline \multicolumn{3}{|l|}{ Ethnicity } \\
\hline White British & $7(35)$ & $9(45)$ \\
\hline White: Irish and other white & $3(15)$ & $6(30)$ \\
\hline Mixed & $3(15)$ & $1(5)$ \\
\hline Asian: Indian, Pakistani, Bangladeshi and other Asian & $2(10)$ & 0 \\
\hline Black Caribbean and Black African & $2(10)$ & $2(10)$ \\
\hline Chinese & $1(5)$ & 0 \\
\hline Other/prefer not to answer & $2(10)$ & $2(10)$ \\
\hline \multicolumn{3}{|l|}{ PCOS phenotype* } \\
\hline $\begin{array}{l}\text { Type A: menstrual disturbances, hyperandrogenism and } \\
\text { polycystic ovaries }\end{array}$ & $15(75)$ & $10(50)$ \\
\hline Type B: menstrual disturbances, hyperandrogenism & 0 & $1(5)$ \\
\hline Type D: menstrual disturbances and polycystic ovaries & $5(25)$ & $9(45)$ \\
\hline \multicolumn{3}{|l|}{ Employment status* } \\
\hline Full-time employment & $12(60)$ & $11(55)$ \\
\hline Part-time employment & $2(10)$ & $3(15)$ \\
\hline Self-employed & $2(10)$ & $2(10)$ \\
\hline Student & $1(5)$ & $3(15)$ \\
\hline Homemaker & $1(5)$ & $1(5)$ \\
\hline Out of work, looking for work & $2(10)$ & 0 \\
\hline
\end{tabular}

*Not compared at baseline.

CAM, complementary and alternative medicine, defined as prior use of one or more CAM treatments including Chinese herbal medicine, acupuncture, Western herbal medicine, nutritional supplements, homeopathy, aromatherapy, reiki healing, hypnotherapy, osteopathy, chiropractic or other (request to specify).

$\mathrm{CHM}$, Chinese herbal medicine.

8 (20\%) withdrew and $3(7.5 \%)$ were lost to follow-up. Details of withdrawals and loss to follow-up are provided in later sections.

\section{Clinical data}

Primary outcome: menstrual regularity at 6 months

Insufficient menstrual data at baseline and at end of study meant it was not possible to compare mean and variability of cycle lengths as our predefined assessment of menstrual regularity. We subsequently followed methods published in other PCOS studies by calculating a menstrual rate per 28 days and by dichotomising to responders and non-responders. ${ }^{32} 33$ Clinically, this means that normal menstrual rate of 9-12 menstrual cycles per year equates to a menstrual rate of $0.75-1$ per month, oligomenorrhoea defined as 3-8 menstrual cycles per year equates to $0.25-0.67$ per month, and amenorrhoea as being $<0.25$ per month.

We analysed menstrual data from 19 participants on standardised CHM and 18 participants on individualised CHM. Data were unavailable from three participants who withdrew or were lost to follow-up within 21 days postrandomisation.

ANCOVA of mean menstrual rate at 6 months, adjusting for baseline menstrual rate and age, was not statistically significant between group (mean difference (MD) $0.10 \pm 0.08,95 \%$ CI -0.07 to $0.26 ; \mathrm{p}=0.26$ ). Statistically significant changes in menstrual rates were seen within group for both standardised CHM (MD $0.18 \pm 0.06$, 95\% CI 0.06 to $0.29 ; \mathrm{p}=0.0027$ ) and individualised CHM (MD $0.27 \pm \mathrm{SE} 0.06,95 \%$ CI 0.15 to 0.39; $\mathrm{p}<0.001$; table 3). 
Figure 1 CONSORT participant flow chart. ALT, alanine aminotransferase.

\section{Enrolment}

Assessed for eligibility ( $n=245$ )

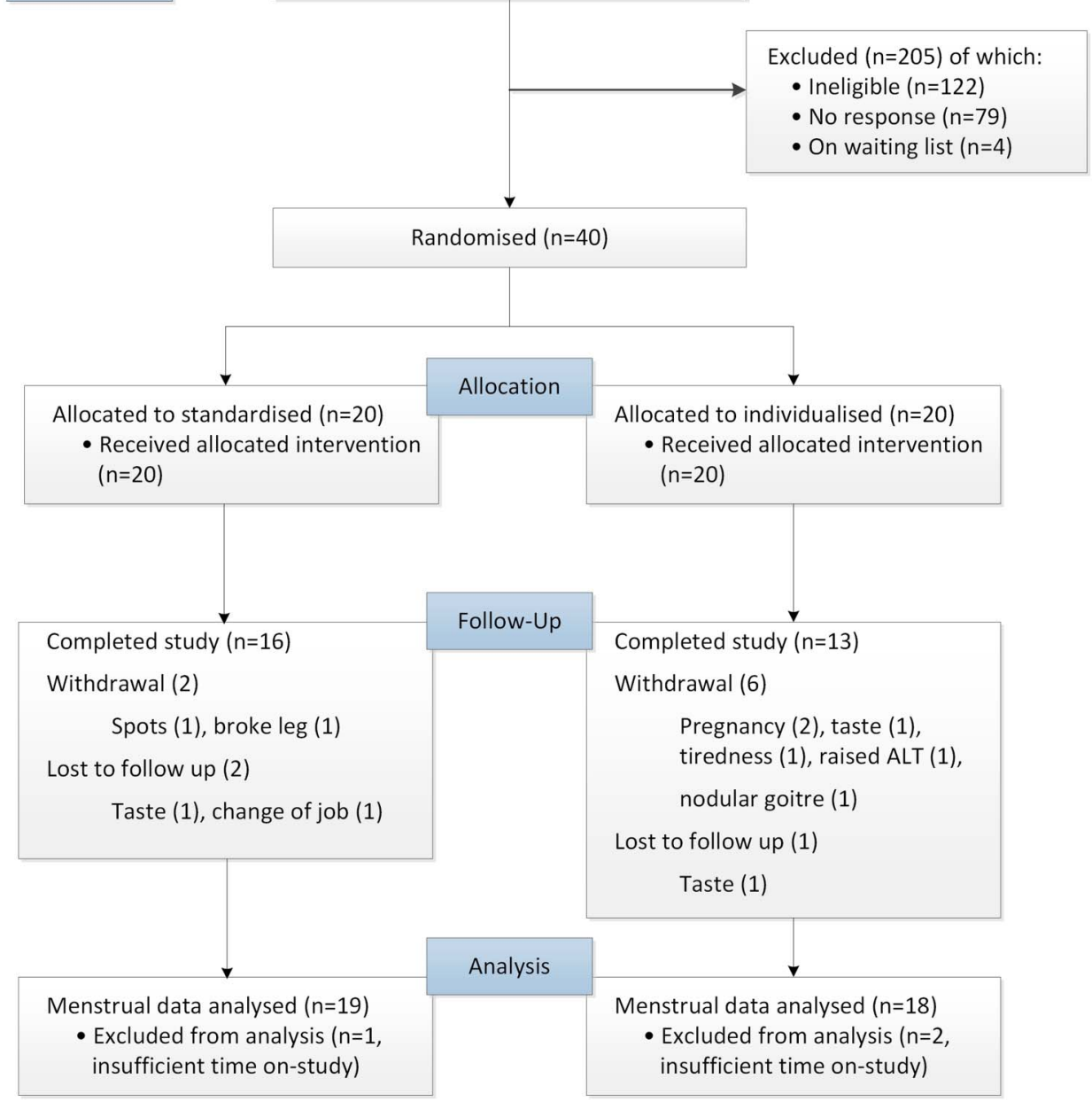

Table 3 Menstrual rate at baseline and 6 months

\begin{tabular}{|c|c|c|c|c|c|c|}
\hline \multirow[b]{3}{*}{ Assessment } & \multicolumn{6}{|c|}{ Menstrual rate (menses/28 days) } \\
\hline & \multicolumn{2}{|c|}{ Standardised group } & \multicolumn{2}{|c|}{$\begin{array}{l}\text { Individualised } \\
\text { group }\end{array}$} & \multicolumn{2}{|l|}{ Between group } \\
\hline & $\mathbf{n}$ & $\begin{array}{l}\text { Mean } \pm \text { SE } \\
(95 \% \mathrm{Cl})\end{array}$ & $\mathbf{n}$ & $\begin{array}{l}\text { Mean } \pm \text { SE } \\
(95 \% \mathrm{Cl})\end{array}$ & $\begin{array}{l}\text { Adjusted mean } \\
\text { difference } \pm S E \\
(95 \% \mathrm{Cl})\end{array}$ & p Value \\
\hline Baseline & 20 & $\begin{array}{l}0.38 \pm 0.06 \\
(0.25 \text { to } 0.52)\end{array}$ & 20 & $\begin{array}{l}0.51 \pm 0.06 \\
(0.38 \text { to } 0.64)\end{array}$ & NA & \\
\hline 6 months menstrual rate* & 19 & $\begin{array}{l}0.62 \pm 0.06 \\
(0.50 \text { to } 0.73)\end{array}$ & 18 & $\begin{array}{l}0.71 \pm 0.06 \\
(0.60 \text { to } 0.83)\end{array}$ & $\begin{array}{l}0.10 \pm 0.08 \\
(-0.07 \text { to } 0.26)\end{array}$ & 0.26 \\
\hline Change from baseline at 6 months ${ }^{*}$ & 19 & $\begin{array}{l}0.18 \pm 0.06 \\
(0.06 \text { to } 0.29)\end{array}$ & 18 & $\begin{array}{l}0.27 \pm 0.06 \\
(0.15 \text { to } 0.39)\end{array}$ & & \\
\hline
\end{tabular}

We dichotomised menstrual data into responders and non-responders by defining response as a change from amenorrhoea to either oligomenorrhoea or eumenorrhoea, a change from oligomenorrhoea to eumenorrhoea, or as achieving pregnancy. Comparing response rates between standardised group $(n=10$ of 19 , $52.6 \%)$ versus individualised group $(n=13$ of $18,72.3 \%)$, this did not reach statistical significance between group (OR 1.37, 95\% CI 0.82 to $2.29, \mathrm{p}=0.22$ ).

Secondary outcomes

ANCOVA of prespecified secondary measures, adjusting for baseline measures and age, were used to compare between-group differences (table 4). While our main 
Table 4 ANCOVA comparing secondary outcome measures within and between group

\begin{tabular}{|c|c|c|c|c|c|c|c|}
\hline \multirow{2}{*}{$\begin{array}{l}\text { Outcome measure } \\
\text { by group }\end{array}$} & \multirow[b]{2}{*}{$\mathbf{n}$} & \multirow{2}{*}{$\begin{array}{l}\text { Adjusted mean } \\
\text { difference from } \\
\text { baseline }\end{array}$} & \multirow[b]{2}{*}{ SE } & \multicolumn{2}{|l|}{$95 \% \mathrm{Cl}$} & \multirow[b]{2}{*}{$p$ Value } & \multirow[b]{2}{*}{$\begin{array}{l}p \text { Value } \\
\text { between group }\end{array}$} \\
\hline & & & & Lower limit & Upper limit & & \\
\hline \multicolumn{8}{|c|}{ Modified Ferriman-Gallwey score } \\
\hline Standardised & 15 & -2.52 & 0.42 & -3.40 & -1.65 & $<0.001$ & \multirow[t]{2}{*}{0.09} \\
\hline Individualised & 14 & -1.44 & 0.44 & -2.35 & -0.54 & 0.001 & \\
\hline \multicolumn{8}{|l|}{ BMI $\left(\mathrm{kg} / \mathrm{m}^{2}\right)$} \\
\hline Standardised & 15 & -0.41 & 0.46 & -1.40 & 0.54 & 0.37 & \multirow[t]{2}{*}{0.83} \\
\hline Individualised & 15 & -0.26 & 0.48 & -1.25 & 0.72 & 0.59 & \\
\hline \multicolumn{8}{|l|}{ Weight $(\mathrm{kg})$} \\
\hline Standardised & 15 & -1.13 & 1.30 & -3.80 & 1.54 & 0.38 & \multirow[t]{2}{*}{0.85} \\
\hline Individualised & 14 & -0.77 & 1.34 & -3.53 & 1.99 & 0.57 & \\
\hline \multicolumn{8}{|l|}{ Waist-to-hip ratio } \\
\hline Standardised & 15 & 0.002 & 0.01 & -0.02 & 0.02 & 0.84 & \multirow[t]{2}{*}{$0.02^{*}$} \\
\hline Individualised & 12 & -0.04 & 0.01 & -0.06 & -0.01 & $<0.001$ & \\
\hline \multicolumn{8}{|c|}{ Waist circumference (cm) } \\
\hline Standardised & 15 & -0.80 & 1.28 & -3.45 & 1.85 & 0.53 & \multirow[t]{2}{*}{0.27} \\
\hline Individualised & 14 & -2.96 & 1.38 & -5.81 & -0.11 & 0.03 & \\
\hline \multicolumn{8}{|l|}{ MYMOP1 } \\
\hline Standardised & 17 & $2.04 \dagger$ & 0.39 & 1.24 & 2.84 & $<0.001$ & \multirow[t]{2}{*}{0.98} \\
\hline Individualised & 18 & $2.02 \dagger$ & 0.38 & 1.24 & 2.80 & $<0.001$ & \\
\hline \multicolumn{8}{|l|}{ MYMOP2 } \\
\hline Standardised & 17 & 0.87 & 0.39 & 0.07 & 1.67 & 0.03 & 0.29 \\
\hline Individualised & 18 & 1.46 & 0.38 & 0.68 & 2.24 & $<0.001$ & \\
\hline MYMOP activity & & & & & & & \\
\hline Standardised & 11 & $2.38 \dagger$ & 0.43 & 1.49 & 3.26 & $<0.001$ & 0.19 \\
\hline Individualised & 15 & 1.59 & 0.36 & 0.84 & 2.34 & $<0.001$ & \\
\hline MYMOP well-being & & & & & & & \\
\hline Standardised & 16 & 0.92 & 0.32 & 0.28 & 1.57 & 0.004 & 0.97 \\
\hline Individualised & 18 & 0.90 & 0.30 & 0.30 & 1.51 & 0.003 & \\
\hline PCOSQ total & & & & & & & \\
\hline Standardised & 17 & 5.17 & 1.0 & 3.09 & 7.25 & $<0.001$ & 0.33 \\
\hline Individualised & 18 & 3.74 & 1.00 & 1.72 & 5.77 & $<0.001$ & \\
\hline PCOSQ emotions & & & & & & & \\
\hline Standardised & 17 & $0.97 \dagger$ & 0.22 & 0.53 & 1.42 & $<0.001$ & 0.77 \\
\hline Individualised & 18 & $1.06 \dagger$ & 0.21 & 0.63 & 1.49 & $<0.001$ & \\
\hline PCOSQ body hair & & & & & & & \\
\hline Standardised & 17 & 0.84 & 0.26 & 0.31 & 1.38 & 0.001 & 0.16 \\
\hline Individualised & 18 & 0.32 & 0.26 & -0.20 & 0.83 & 0.11 & \\
\hline PCOSQ weight & & & & & & & \\
\hline Standardised & 17 & 0.79 & 0.32 & 0.15 & 1.44 & 0.01 & 0.61 \\
\hline Individualised & 18 & 0.56 & 0.31 & -0.06 & 1.19 & 0.07 & \\
\hline$P C O S Q$ infertility & & & & & & & \\
\hline Standardised & 17 & $1.17 \dagger$ & 0.25 & 0.66 & 1.68 & $<0.001$ & 0.26 \\
\hline Individualised & 18 & 0.77 & 0.24 & 0.27 & 1.27 & 0.001 & \\
\hline PCOSQ menstrual & & & & & & & \\
\hline Standardised & 17 & $1.40 \dagger$ & 0.24 & 0.91 & 1.88 & $<0.001$ & 0.28 \\
\hline Individualised & 18 & $1.03+$ & 0.23 & 0.56 & 1.50 & $<0.001$ & \\
\hline DLQI & & & & & & & \\
\hline Standardised & 16 & -1.97 & 0.66 & -3.32 & -0.62 & 0.003 & 0.09 \\
\hline Individualised & 18 & -0.36 & 0.62 & -1.63 & 0.91 & 0.56 & \\
\hline
\end{tabular}

${ }^{*}$ Favouring individualised CHM.

†Achieved MCID.

BMI, body mass index; DLQI, Dermatology Life Quality Index; MCID, minimum clinically important difference; MYMOP, Measure Yourself Medical Outcome Profile; PCOSQ, Polycystic Ovary Syndrome Questionnaire.

analysis focuses on change scores, we have additionally provided baseline and postintervention scores, adjusted for age and baseline scores, in an online supplementary data file for interested readers.

\section{Menstrual regularity at end of study}

Owing to final visit arrangements, participation extended beyond 6 months for 26 participants. The nature of menstrual reporting meant these data 
continued to be collected until their final visit and we conducted a post hoc analysis to explore the effects of this additional data on menstrual rate.

Median days on study for standardised CHM was 175 days (IQR 167-180) and for individualised CHM was 177.5 days (IQR 167-190). As in our primary analysis, between-group differences were not statistically significant (MD $0.10 \pm 0.09,95 \%$ CI -0.09 to $0.29 ; \mathrm{p}=0.27$ ). At final visit, changes from baseline were again statistically significant for standardised CHM (MD 0.25 $\pm 0.06,95 \%$ CI 0.12 to $0.38 ; \mathrm{p}<0.001$ ) and individualised CHM (MD $0.35 \pm 0.07,95 \%$ CI 0.22 to $0.49 ; \mathrm{p}<0.001)$.

\section{Hirsutism}

Statistically significant reductions in $\mathrm{mFG}$ scores from baseline were seen in both standardised group (MD $-2.52 \pm 0.42,95 \%$ CI -3.40 to $-1.65 ; \mathrm{p}<0.001)$ and individualised group (MD $-1.44 \pm 0.44,95 \% \mathrm{CI}-2.35$ to -0.54 ; $\mathrm{p}=0.001$ ) but which did not reach between-group significance $(p=0.09)$. Patient-reported quality of life relating to hirsutism is presented as part of the PCOSQ data in Polycystic Ovary Syndrome Questionnaire section.

\section{Anthropometrics}

Changes observed in BMI and weight were not statistically significant within or between group. Statistically significant changes in waist circumference were apparent within group for individualised CHM (MD $-2.96 \pm 1.38$, $95 \%$ CI -5.81 to $-0.11 ; \mathrm{p}=0.03$ ) but were seen neither in standardised CHM (MD $-0.8 \pm 1.28,95 \%$ CI -3.45 to $1.85 ; \mathrm{p}=0.53$ ), nor between group (MD $-2.16 \pm 1.90,95 \%$ CI -6.08 to $1.75 ; \mathrm{p}=0.27$ ). Individualised CHM also saw statistically significant within-group changes from baseline in WHR; MD $-0.04 \pm 0.01,95 \%$ CI -0.06 to 0.01 ; $\mathrm{p}<0.001$ ) but which were not seen in standardised CHM (MD $0.002 \pm 0.01,95 \%$ CI -0.02 to $0.02 ; \mathrm{p}=0.84$ ). This difference was statistically significant between groups favouring individualised CHM ( $\mathrm{p}=0.02)$.

\section{Measure Yourself Medical Outcome Profile}

Count and frequency ranking of MYMOP1 and MYMOP2 data indicated that the three most distressing concerns were hirsutism $(n=19)$, weight $(n=16)$ and menstrual regularity $(\mathrm{n}=12)$.

For both groups, statistically significant improvements were seen within group for all four MYMOP submeasures of MYMOP1 (standardised $\mathrm{p}<0.001$; individualised $\mathrm{p}<0.001$ ), MYMOP2 (standardised $\mathrm{p}=0.03$; individualised $\mathrm{p}<0.001$ ), MYMOP activity (standardised $\mathrm{p}<0.001$; individualised $\mathrm{p}<0.001$ ) and MYMOP well-being (standardised $p=0.004$; individualised $p=0.003$ ). There were however no between-group differences in any of these measures (MYMOP1 $\mathrm{p}=0.98$, MYMOP2 $\mathrm{p}=0.29$, MYMOP activity $\mathrm{p}=0.19$, MYMOP well-being $\mathrm{p}=0.97$ ). Minimum clinically important difference (MCID) of 1.0 was reached for MYMOP1 for both groups and for MYMOP activity for only standardised CHM. All remaining MYMOP results show potential for a clinically important difference since 95\% CIs include the MCID.

\section{Polycystic Ovary Syndrome Questionnaire}

For both groups, statistically significant improvements were seen in PCOS total scores within group for both standardised CHM (MD 5.17 $\pm 1.0,95 \%$ CI 3.09 to 7.25; $\mathrm{p}<0.001$ ) and individualised CHM (MD 3.74 $\pm 1.0,95 \%$ CI 1.72 to $5.77 ; \mathrm{p}<0.001)$ but not between group $(p=0.33)$. Statistically significant improvements were seen within group for all PCOSQ domains for standardised CHM for emotions $(p<0.001)$, body hair ( $p=0.001)$, weight $(p=0.01)$, infertility $(p<0.001)$ and menstrual $(p<0.001)$; and for individualised CHM for emotions $(p<0.001)$, infertility $(p=0.0010)$ and menstrual $(\mathrm{p}<0.001)$ domains only. No between-group differences were detected. The MCID of 0.5 was reached for emotions and menstrual domains for both groups, and for infertility in only the standardised group. All remaining PCOSQ domain scores show potential for a clinically important difference.

\section{Dermatology Life Quality Index}

A statistically significant within-group change in DLQI score was seen for standardised treatment $(p=0.003)$ but not in individualised treatment $(\mathrm{p}=0.56)$ and which was not statistically significant between group $(\mathrm{p}=0.09)$. MCID of 3.2 was not met in either group but the $95 \%$ CI for standardised CHM includes 3.2, suggesting potential for clinical benefit.

\section{Safety and adverse events}

Liver and kidney function

The vast majority of ALT and creatinine tests were normal for both treatments. However, at week 4, one participant in individualised CHM displayed normal creatinine but abnormal ALT readings and was withdrawn as a precautionary measure. This was later assessed as being alcohol related and not related to study medication.

\section{Nature and duration of adverse events and adverse reactions}

Adverse reactions (AR) were minor with nine reactions reported among six participants in standardised CHM and four participants in individualised CHM. All nine were assessed as mild in severity, expected, not serious and having a reasonable causal relationship with treatment. Gastrointestinal symptoms (bloating, nausea, loose stools, vomiting) were the most commonly reported with the remainder consisting of tiredness, skin breakout, ovulation pain, light headedness and headache.

The majority of AR incidents $(14 / 17,82.4 \%)$ subsided with continued administration and lasted a median of 4 days (range 1-74 days). Two symptoms (loose stools and bloating) persisted with continued administration of standardised CHM for two participants. 
Two serious adverse events were reported. One participant allocated to individualised CHM was diagnosed with nodular goitre and one participant allocated to standardised CHM experienced a leg fracture. Causality for both events was assessed by hospital consultants independent of the study and confirmed to be unrelated to study treatment.

\section{Pregnancy}

Two participants taking individualised CHM became pregnant on study and were withdrawn for monitoring. Both reported healthy pregnancies and uncomplicated live births at full term.

\section{Process evaluation}

\section{Blinding}

BBIs were calculated from practitioner blinding data at weeks $4(n=36)$ and $12(n=31)$ and final visit $(n=36)$ following published methods. ${ }^{31} 34$ At week 4, this was standardised (BBI $-0.11,95 \%$ CI -0.35 to 0.14 , random) and individualised (BBI $0.47,95 \%$ CI 0.23 to 0.71 , unblinded); week 12 standardised (BBI $-0.24,95 \%$ CI -0.54 to 0.07 , random) and individualised (BBI 0.50, $95 \%$ CI 0.12 to 0.88 , unblinded); and final visit standardised (BBI $-0.56,95 \%$ CI -0.91 to -0.20 , opposite) and individualised (BBI 0.61, 95\% CI 0.30 to 0.92, unblinded). The most commonly cited reasons for treatment guesses were 'presence of effects' and 'lack of effects' which consistently led to guesses of individualised and standardised CHM, respectively.

\section{Security of randomisation and allocation procedures}

Randomisation, allocation and CHM dispatch were conducted as intended, after comparing the randomisation list from our statistician against the randomisation $\log$ generated by the pharmacy and a sample of 89 (39.9\%) prescriptions.

\section{Adherence}

Both groups demonstrated a small increase in MMAS mean scores by the final visit (standardised MD $0.9 \pm \mathrm{SD}$ 2.2, $95 \%$ CI -0.3 to 2.0; individualised MD 1.0 $1.4,95 \%$ CI 0.3 to 1.8 ) which was not statistically significant between group $(\mathrm{p}=0.86)$. A mean of $65 \%$ (SD 21.2) of CHMs had been administered, calculated from 21 participants $(52.5 \%)$ for whom at least $75 \%$ of prescription data had been accounted for.

\section{Participant and CRF evaluation}

Overall, participants reported positive experiences of the study. Thirty-two participants $(80 \%)$ reported they would 'definitely' or 'probably' agree to take part in a study like this again. Three participants $(7.5 \%)$ reported being 'unsure' or 'probably not'. There were five nonresponses $(12.5 \%)$.

Thematic analysis of participant feedback highlighted five broad themes: (1) appraisal of the CHM intervention, (2) managing practicalities of research participation, (3) empowerment and enablement, (4) management of care and (5) quality of care. Participants appraised CHM by improvements in symptoms of personal concern and not necessarily menstrual regularity. Participants raised concerns regarding safety and taste difficulties that reduced adherence and described feeling empowered through gaining further knowledge of PCOS and of CHM within this study. Aspects of care management were highly valued such as frequent monitoring and a holistic and personalised approach. Quality of care was appraised positively and seen to be delivered in an approachable, non-judgemental and knowledgeable manner, and with compassion and trust.

To evaluate study processes, we analysed thematically CRF data which highlighted three broad themes: (1) improving participant experience, (2) improving trial conduct and management, and (3) design considerations for future research. Highlighted areas for improvement suggested improved consideration for participant anxieties surrounding taking anthropometric and hirsutism assessments. Management of CHMs was considered complex, particularly surrounding CHM administration and weighing which could have been discussed in more detail with participants. Participants reported wishing to finding out personal and overall study progress and recommended the inclusion of biochemical investigations and ovarian status in future research. Limitations with hirsutism assessment were raised as it did neither consider rate of hair growth nor area of hair distribution. Future work could consider frequency of hair removal or time until hair regrowth which may reflect more accurately hirsutism status as appraised by participants.

\section{DISCUSSION}

The findings of this study suggest that it is feasible to conduct a clinical study in the UK using an RCHM-approved dispensary, offering either individualised or standardised multiherb CHM treatment for 6 months to women with PCOS-related oligomenorrhoea and amenorrhoea. Our results support the evaluation of oligomenorrhoea and amenorrhoea, but suggest that hirsutism could be considered as a primary outcome since this was a key concern for our participants according to our MYMOP1 and MYMOP2 ranking data. Individualised and standardised CHMs when prescribed by a registered CHM practitioner and administered for 6 months appear to be safe, without harmful effects on either liver or kidney function, and with minimal side effects. Although these findings appear encouraging, they should be considered preliminary owing to the sample size and the feasibility nature of this study and our results relating to clinical effects and safety should be interpreted with these limitations in mind.

Our preliminary data support the use of either individualised or standardised CHM as there was no statistically significant difference between group in 
improvement of menstrual rate at 6 months. However, we acknowledge that this feasibility study had a small sample size and we cannot rule out the possibility that a larger sample would provide greater power to detect a potentially important difference. Although we considered the retention rate of $72.5 \%$ acceptable, changing administration to once per day and to a tablet or capsule are likely to further improve participant retention. Finally, we were unsuccessful in recruiting CHM practitioners and would recommend that a future study provide financial reimbursement for practitioner time and to plan for better access to safety testing.

For this study, we were unable to include a control arm such as placebo, wait list or treatment as usual and our results should be interpreted with caution owing to factors that were not controlled for such as contextual effects and regression to the mean. We however successfully evaluated feasibility as planned and a significant strength of this study is that we collected data prospectively on standardised and individualised multiherb CHMs which were administered according to real-world practice and for 6 months. Safety and adverse events data from our study appear to be consistent with the findings of previous CHM studies in the $\mathrm{UK}^{29} 3536$ which suggest that CHMs appear to be safe and overall well tolerated. Our study compared real-world CHM-prescribing approaches for a significant duration of time, whereas the majority of previous PCOS and CHM trials evaluate standardised CHMs for $<6$ months. ${ }^{18} 37$ Although our safety data are encouraging and appears consistent with the findings from an Australian survey, ${ }^{38}$ this was a small-scale study and larger studies with longer term follow-up remain warranted. Owing to financial constraints, we were unable to conduct biochemical tests and evaluate polycystic ovarian morphology as part of our outcome measures and we would strongly recommend including these in a main study to increase the rigour of the study design and to maximise relevance of such findings for clinicians and for the PCOS population. Future feasibility studies should consider including these PCOS-relevant medical investigations to increase scientific rigour.

We successfully recruited the target sample size and found an acceptable retention rate of $72.5 \%$. This suggests strong interest from the PCOS population in the UK which is important since less than one-third of primary care trials are able to recruit within the specified timeframe ${ }^{39}$ and high attrition rates of up to $53 \%$ are typically found in PCOS studies. ${ }^{40}$ 41 However, we recruited outside of primary care and participants were aware they would be randomised to one of two active CHM treatments. A question that remains unanswered is the recruitment and retention rate within a National Health Service (NHS) setting and the willingness of participants to be randomised to a placebo or wait-list control group. Furthermore, it is possible that we recruited a selective group since a significant proportion of our participants reported previously discontinuing conventional medicines due to side effects or lack of effects, and had prior use of CAM and of Chinese medicine. However, we argue that this could potentially reflect real-world prescribing strategies where women resistant to first-line treatments such as the contraceptive pill or insulin-sensitising agents could instead be offered as therapeutic alternatives such as CHMs, provided there was robust evidence for clinical effect and safety.

Although evaluating oligomenorrhoea and amenorrhoea would be an appropriate primary outcome measure for the main trial, this feasibility study has highlighted challenges in statistical and clinical interpretation of menstrual regularity. Insufficient menstrual data at baseline and at end of study meant it was not possible to use our predefined approach of assessing menstrual regularity and this was thus evaluated as a menstrual rate following other PCOS studies. ${ }^{25} 3233$ Although this enables our results to be more easily compared with the wider literature, we acknowledge this was a decision made post hoc. Furthermore, it is possible that our baseline menstrual diary data were subject to recall bias and which could be overcome by considering a run-in phase. As hirsutism ranked higher than menstrual irregularity as a key concern for participants in the MYMOP data, it is possible that evaluating hirsutism would be more relevant to patients with PCOS and which in turn could facilitate recruitment in a future study. This needs to be considered alongside shortcomings of hirsutism assessment we have already highlighted, suggesting that a subjective or quality of life measure may be more appropriate.

Robust evaluation of adherence and practitioner blinding is important in RCTs as a means of assessing internal validity. We found the process of collecting both self-reported and objective adherence data useful to triangulate findings and would recommend this for future trials. These data enabled us to conclude that a reduction in prescribed CHM dosage may be appropriate to improve participant acceptability and without compromising clinical effect on menstrual cyclicity. By close of study, our practitioner blinding results point to a 'standardised opposite and individualised unblinded' scenario, suggesting that blinding was likely secure. This highlights a 'wishful thinking' scenario that is consistent with other studies and suggests a high response bias, where the desire to believe that the more effective intervention-in this case the individualised treatment-was associated with a treatment response. ${ }^{31} 42$ This emphasises the importance of blinding caregivers and assessors in RCTs and in evaluating blinding as a key factor in internal validity.

\section{CONCLUSIONS}

We have demonstrated that it is feasible to conduct a CHM trial for oligomenorrhoea and amenorrhoea in PCOS. Our preliminary findings suggest that individualised and standardised multiherb CHMs have similar safety profiles and clinical effects on menstrual regularity. This evaluation supports the use of menstrual 
regularity as a primary outcome measure for a main study which should include an appropriate control group such as placebo or treatment as usual.

\section{Author affiliations}

${ }^{1}$ University of Southampton Primary Care and Population Sciences Aldermoor Health Centre Aldermoor Close, Southampton, UK

${ }^{2}$ University of Southampton, Mathematical Sciences Highfield Southampton, Southampton, UK

${ }^{3}$ Bournemouth University Centre for Ultrasound Studies, Bournemouth, UK

\section{Twitter Follow Lily Lai @lilylai}

Acknowledgements The authors are grateful to George $\mathrm{He}$ and the Phoenix Medical team for CHM dispensing support and for discounted CHMs. The authors would like to thank all participants for their time and contribution to this study, without whom this work would not have been possible.

Contributors All authors have made important and impactful contributions to the present manuscript. LL is the primary author of this research study and was granted the funding to conduct this work as part of her research training fellowship. She has made substantial contributions to the study design, acquisition of data, analysis and interpretation and in writing and preparing the manuscript. AF made a substantial contribution to the concept and design of this research and provided mentorship throughout the process. PP has provided statistical guidance throughout the project with input into randomisation, data collection and planned and eventual data analysis. TW has made important contributions to the design of this research study and supporting recruitment and screening of participants. MM has had an integral role in this project by providing guidance in study design, recruitment of participants and providing critical comments on data interpretation and manuscript preparation. GL has made a substantial contribution to this project by providing mentorship and guidance in study concept, design, participant eligibility and data interpretation and manuscript preparation. All authors have approved of the final version of this manuscript.

Funding This study is supported by a PhD research training fellowship (RCS Ref 10-0894) awarded to the main author by the National Institute for Health Research (NIHR) School for Primary Care Research.

Disclaimer The views expressed are those of the authors and not necessarily those of the NHS, the NIHR or the Department of Health. Other than the role stated, those named have had no other contribution to the study design, analysis or writing of the protocol and of this report.

\section{Competing interests None declared.}

\section{Patient consent Obtained.}

Ethics approval Ethical approval was granted in December 2012 from the University of Southampton Faculty of Medicine Research Ethics Committee; Register of Chinese Herbal Medicine Research Ethics Committee (Approvals ID 3977).

Provenance and peer review Not commissioned; externally peer reviewed.

Data sharing statement No additional data are available.

Open Access This is an Open Access article distributed in accordance with the terms of the Creative Commons Attribution (CC BY 4.0) license, which permits others to distribute, remix, adapt and build upon this work, for commercial use, provided the original work is properly cited. See: http:// creativecommons.org/licenses/by/4.0/

\section{REFERENCES}

1. Asunción M, Calvo RM, San Millán JL, et al A prospective study of the prevalence of the polycystic ovary syndrome in unselected Caucasian women from Spain. J Clin Endocrinol Metab 2000;85:2434-8. http://dx.doi.org/10.1210/jcem.85.7.6682

2. March WA, Moore VM, Willson KJ, et al. The prevalence of polycystic ovary syndrome in a community sample assessed under contrasting diagnostic criteria. Hum Reprod 2010;25:544-51.
3. Ehrmann DA. Polycystic ovary syndrome. N Engl J Med 2005;352:1223-36.

4. Balen AH, Conway GS, Kaltsas G, et al. Polycystic ovary syndrome: the spectrum of the disorder in 1741 patients. Hum Reprod 1995;10:2107-11.

5. Vutyavanich T, Khaniyao V, Wongtra-Ngan S, et al. Clinical, endocrine and ultrasonographic features of polycystic ovary syndrome in Thai women. J Obstet Gynaecol Res 2007;33:677-80.

6. Sills ES, Perloe M, Tucker MJ, et al. Diagnostic and treatment characteristics of polycystic ovary syndrome: descriptive measurements of patient perception and awareness from 657 confidential self-reports. BMC Womens Health 2001;1:3.

7. Fleming R, Hopkinson ZE, Wallace AM, et al. Ovarian function and metabolic factors in women with oligomenorrhea treated with metformin in a randomized double blind placebo-controlled trial. J Clin Endocrinol Metab 2002;87:569-74.

8. Donnan PT, MacDonald TM, Morris AD. Adherence to prescribed oral hypoglycaemic medication in a population of patients with type 2 diabetes: a retrospective cohort study. Diabet Med 2002;19:279-84.

9. Grant RW, Devita NG, Singer DE, et al. Polypharmacy and medication adherence in patients with type 2 diabetes. Diabetes Care 2003;26:1408-12.

10. Legro RS, Barnhart HX, Schlaff WD, et al. Clomiphene, metformin, or both for infertility in the polycystic ovary syndrome. N Engl J Med 2007;356:551-66.

11. Arentz S, Smith CA, Abbott JA, et al. A survey of the use of complementary medicine by a self-selected community group of Australian women with polycystic ovary syndrome. BMC Complement Altern Med 2014;14:472.

12. Rooney S, Pendry B. Phytotherapy for polycystic ovarian syndrome: a review of the literature and evaluation of practitioners' experiences. $J$ Herbal Med 2014;4:159-71.

13. Ushiroyama T, Hosotani T, Mori K, et al. Effects of switching to wen-jing-tang (unkei-to) from preceding herbal preparations selected by eight-principle pattern identification on endocrinological status and ovulatory induction in women with polycystic ovary syndrome. Am J Chin Med 2006;34:177-87.

14. Takeuchi T, Nishii O, Okamura T, et al. Effect of traditional herbal medicine, shakuyaku-kanzo-to on total and free serum testosterone levels. Am J Chin Med 1989;17:35-44.

15. Heibashy MIA, Mazen GMA, SHahin MI. Metabolic changes and hormonal disturbances in polycystic ovarian syndrome rats and the amelioration effects of metformin and/or cinnamon extraction. J Am Sci 2013;9:54-62.

16. Raja-Khan N, Stener-Victorin E, Wu X, et al. The physiological basis of complementary and alternative medicines for polycystic ovary syndrome. Am J Physiol Endocrinol Metab 2011;301:E1-10.

17. Li XP, Ye S, Lin S. Effects of modified Zi Kui prescription on polycystic ovary syndrome-related hyperandrogenism in liver and kidney yin deficiency syndrome. Guangming Tradit Chin Med 2011;26:242-4.

18. Hao HN. Combined treatment of Huatan Bushen Tang and Diane-35 in 30 cases of polycystic ovary syndrome-related infertility. Henan Tradit Chin Med 2010;30:783-4.

19. Zhou YH, Huang YJ, Liu W, et al. Clinical research on combined therapy of traditional Chinese and Western medicine on polycystic ovarian syndrome. Liaoning J Tradit Chin Med 2010;37:1328-30.

20. Kort DH, Lobo RA. Preliminary evidence that cinnamon improves menstrual cyclicity in women with polycystic ovary syndrome: a randomized controlled trial. Am J Obstet Gynecol 2014;211:487. e1-6.

21. Craig $\mathrm{P}$, Dieppe $\mathrm{P}, \mathrm{Macintyre} \mathrm{S}$, et al. Developing and evaluating complex interventions: the new Medical Research Council guidance. BMJ 2008;337:a1655.

22. Lancaster GA, Dodd S, Williamson PR. Design and analysis of pilot studies: recommendations for good practice. J Eval Clin Pract 2004; 10:307-12.

23. Arain M, Campbell MJ, Cooper CL, et al. What is a pilot or feasibility study? A review of current practice and editorial policy. BMC Med Res Methodol 2010;10:67.

24. Thabane L, Ma J, Chu R, et al. A tutorial on pilot studies: the what, why and how. BMC Med Res Methodol 2010;10:1.

25. Lai LYW, Flower A, Moore M, et al. Polycystic ovary syndrome: a randomised feasibility and pilot study using Chinese Herbal medicine to explore Impact on Dysfunction (ORCHID)—study protocol. Eur J Integr Med 2014;6:392-9.

26. Maciocia M. The foundations of Chinese medicine: a comprehensive text for acupuncturists and herbalists. Edinburgh: Elsevier Churchill Livingstone, 1997.

27. Lai L, Flower A, Moore M, et al. Developing clinical practice guidelines for Chinese herbal treatment of polycystic ovary 
syndrome: a mixed-methods modified Delphi study. Complement Ther Med 2015;23:430-8.

28. Rotterdam ESHRE/ASRM-Sponsored PCOS consensus workshop group. Revised 2003 consensus on diagnostic criteria and long-term health risks related to polycystic ovary syndrome (PCOS). Hum Reprod 2004;19:41-7.

29. Flower A, Lewith GT, Little P. A feasibility study exploring the role of Chinese herbal medicine in the treatment of endometriosis. J Altern Complement Med 2011;17:691-9.

30. Yasmin E, Glanville J, Barth J, et al. Effect of dose escalation of metformin on clinical features, insulin sensitivity and androgen profile in polycystic ovary syndrome. Eur J Obstet Gynecol Reprod Biol 2011;156:67-71.

31. Enblom A, Johnsson A, Hammar M, et al. The nonpenetrating telescopic sham needle may blind patients with different characteristics and experiences when treated by several therapists. Evid Based Complement Alternat Med 2011;2011:185034.

32. Moghetti P, Castello R, Negri C, et al. Metformin effects on clinical features, endocrine and metabolic profiles, and insulin sensitivity in polycystic ovary syndrome: a randomized, double-blind,

placebo-controlled 6-month trial, followed by open, long-term clinical evaluation. J Clin Endocrinol Metab 2000;85:139-46.

33. Essah PA, Apridonidze T, luorno MJ, et al. Effects of short-term and long-term metformin treatment on menstrual cyclicity in women with polycystic ovary syndrome. Fertil Steril 2006;86:230-2.
34. Bang $\mathrm{H}, \mathrm{Ni} \mathrm{L}$, Davis $\mathrm{CE}$. Assessment of blinding in clinical trials. Control Clin Trials 2004;25:143-56.

35. Sheehan MP, Atherton DJ. A controlled trial of traditional Chinese medicinal plants in widespread non-exudative atopic eczema. Br J Dermatol 1992;126:179-84.

36. Scheid V, Tuffrey V, Weijburg $T$, et al. Chinese medicine treatment for menopausal symptoms in the UK health service: is a clinical trial warranted? Maturitas 2015;80:179-86.

37. Li XB, Zhai JL, Li LY, et al. Influence of Lingzhu granules on endocrine and lipid metabolism of polycystic ovary syndrome patients. J Tradit Chinese Med 2011;52:32-4.

38. Bensoussan A, Myers SP, Carlton AL. Risks associated with the practice of traditional Chinese medicine: an Australian study. Arch Fam Med 2000;9:1071-8.

39. Bower P, Wilson S, Mathers N. Short report: how often do UK primary care trials face recruitment delays? Fam Pract 2007;24:601-3.

40. Sørensen LB, Søe M, Halkier KH, et al. Effects of increased dietary protein-to-carbohydrate ratios in women with polycystic ovary syndrome. Am J Clin Nutr 2012;95:39-48.

41. Moran LJ, Hutchison SK, Norman RJ, et al. Lifestyle changes in women with polycystic ovary syndrome. Cochrane Database Syst Rev 2011;(7):CD007506.

42. Moroz A, Freed B, Tiedemann L, et al. Blinding measured: a systematic review of randomized controlled trials of acupuncture. Evid Based Complement Alternat Med 2013;2013:708251. 